\title{
Manejo de quiste dentígero mandibular más fijación ósea rígida. Presentación de un caso
}

\author{
Managment of the mandibular dentigerous cyst plus rigid bone fixation, Presentation of a case

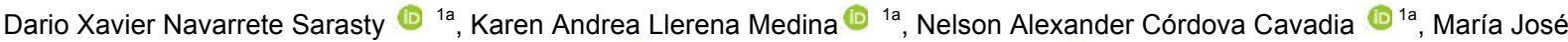 \\ Jarrín Peñafiel ${ }^{10}{ }^{1 a}$, Jaime Arturo Castro Pallaroso 당 ${ }^{1 b}$
}

\section{RESUMEN}

El objetivo de este reporte es mostrar el manejo de un paciente con diagnóstico de quiste dentígero mandibular. Se presenta el caso de un paciente de sexo masculino de 14 años de edad, sin antecedentes mórbidos de importancia a la clínica odontológica SERODU, en la tomografía axial computarizada se evidenció una sombra radiolúcida entre las piezas dentales 42,44 y 45 con la pieza $N^{\circ} 43$ incluida en la sínfisis de la región mandibular, posición horizontal de la pieza dental, la corona hacia distal y su raíz hacia medial con formación completa. A nivel de la corona se puede observar una sombra radiolúcida bien definida con un halo radiopaco, compatible con un quiste dentígero. Por lo que se decidió llevar acabo el tratamiento endodóntico en las piezas dentales $43,44,45$, posteriormente se realizó la enucleación de la lesión, exéresis de la pieza dentaria, curetaje y fijación óseo rígida, para evitar complicaciones patológicas de estructuras adyacentes como fractura mandibular por la dimensión de la lesión. Se obtuvo un diagnóstico histopatológico de quiste dentígero. Al control de treinta días, se observó radiográficamente, una zona radiopaca compatible con regeneración ósea, con un halo radiolúcido de separación con el hueso basal original. La enucleación y la fijación ósea rígida, resultaron ser un tratamiento eficaz para eliminar dicha lesión que resultó ser benigna.

Palabras Clave: Diente impactado; Quiste dentígero; Placas Óseas; Tomografía Axial Computarizada. (Fuente: DeCS BIREME)

\begin{abstract}
The objective of this article is to present the management of a patient diagnosed with a mandibular dentigerous cyst. The present case deals with a 14-year-old male patient, without relevant antecedents who attended the SERODU dental clinic, a radiolucent shadow between the dental pieces 42,44 and 45 was included in the computerized axial tomography with the part piece No. 43 included in the symphysis of the mandibular region, horizontal position of the dental piece, the crown towards the distal and its root towards the medial with complete formation. At the crown level, a well-defined radiolucent shadow can be observed with a radiopaque halo, compatible with a dentigerous cyst. Therefore, it was decided to carry out the endodontic treatment in the dental pieces $43,44,45$, subsequently the enucleation of the lesion, elimination of the dental piece, curettage and rigid bone fixation were performed, to avoid pathological complications of adjacent structures such as fracture mandibular due to the size of the lesion. A histopathological diagnosis of dentigerous cyst was obtained. At the thirty-day control, a radiopaque area compatible with bone regeneration was observed radiographically, with a radiolucent halo separating with the original basal bone. Enucleation and rigid bone fixation is an effective treatment technique to eliminate the lesion that turned out to be benign.
\end{abstract}

Key words: Tooth Impacted; Dentigerous Cyst; Bone Plates; Tomography. (Source: MeSH NLM)

Recibido: 15 de marzo de 2020

Aprobado: 30 de junio de 2020

Publicado: 6 julio de 2020

\footnotetext{
${ }^{1}$ Universidad Tecnológica de Ecuador (UTE). Ecuador

adontólogo

${ }^{\mathrm{b}}$ Especialista en Cirugía Maxilofacial
}

Este es un artículo Open Access distribuido bajo la licencia Creative Commons Atribución-NoComercialCompartirlgual 4.0

(우@()

Correspondencia:

María José Jarrín

Dirección: Calle Bolívar 6-38 y González Suárez. Ecuador

Correo electrónico: majp_1995@outlook.com

Citar como: Navarrete Sarasty D, Llerena Medina K, Córdova Cavadia N, Jarrín Peñafiel MJ, Castro Pallaroso J. Manejo de quiste dentígero mandibular mas fijación ósea rígida. Presentación de un caso. KIRU. 2020; 17(3): 143-148. https://doi.org/10.24265/kiru.2020.v17n3.05. 


\section{INTRODUCCIÓN}

Se considera que los quistes dentígeros son un conjunto de entidades que pueden tener un origen odontogénico y no odontogénico, y que además pueden ser tanto benignos como malignos (1). Generalmente, se forman en cualquiera de las etapas de desarrollo del epitelio dental ${ }^{(2)}$. Se les define como una bolsa tapizada que esta tapizada interiormente con epitelio y recubierta externamente con tejido conectivo, que pueden tener un contenido líquido o semilíquido ${ }^{(3)}$. No son muy frecuentes pero pueden aparecer mayormente en adultos (4). Estos quistes representan, alrededor, del $35 \%$ del total de quistes mandibulares (5). En su mayoría no muestran síntomas y se descubren en hallazgos radiográficos ${ }^{(6)}$. Es recomendable que el tratamiento sea multidisciplinario, que incluya al cirujano maxilofacial ,al otorrinolaringólogo y al pediatría $^{(7)}$.

De acuerdo a las características de las lesiones el tratamiento puede ser la enucleación, la descompresión la marsupialización ${ }^{(8)}$. Esto dependerá del tamaño y la localización de la lesión, el tipo de dentición, edad del paciente y el compromiso de estructuras anatómicas adyacentes importantes ${ }^{(9)}$.

Se indicara la exéresis si no hubiera riesgo de lesionar estructuras anatómicas, el seno maxilar o el nervio dentario inferior o como ápices dentales vitales cercanos $^{(10)}$.

Por el contrario se indicará la marsupialización si e quiste ha desplazado piezas dentales o se busca favorecer la erupción de un diente impactado ${ }^{(11)}$.

Cuando se habla de enucleación se refiere a la exéresis de la lesión, es decir a removerla despegándola del hueso ${ }^{(12)}$. Esto permite realizar un análisis histopatológico del tejido removido en su totalidad. ${ }^{(13)}$.

El pronóstico de la mayoría de quistes dentígeros es excelente y la recurrencia rara vez es notada después de una completa remoción, varias complicaciones potenciales deben ser consideradas ${ }^{(14)}$. Si las lesiones son grandes, el pronóstico es reservado ya que conllevan una gran pérdida ósea y adelgazan peligrosamente al hueso, existiendo el riesgo de producir fractura patológica del maxilar comprometido ${ }^{(15)}$. Mucho se ha escrito sobre la posibilidad de que el revestimiento del quiste pueda experimentar transformación neoplásica a un ameloblastoma ${ }^{(16)}$.

Las fracturas mandibulares suelen localizarse en regiones que presentan cierta debilidad y en las que la estructura ósea tiene una menor resistencia como el cóndilo mandibular ${ }^{(17)}$ o existe un edentulismo o presencia de dientes retenidos, quistes o largas raíces dentales ${ }^{(18)}$. En el niño los puntos débiles de la mandíbula son la región del germen del canino definitivo, la del segundo molar y el cuello del cóndilo ${ }^{(19)}$.

La técnica de fijación interna rígida se realiza hace aproximadamente 30 años (20), se utilizan dispositivos como placas metálicas y tornillos de compresión, que son suficientemente resistentes como para permitir el uso activo de la estructura esquelética durante la cicatrización ${ }^{(21)}$.

Esta técnica evita la necesidad de utilizar una fijación intermaxilar de las fracturas de tercio medio y mandíbula (22) , además en las fracturas infectadas favorece la cicatrización ósea primaria lo que impide la aparición de callo óseo que podría llevar a un sobre relieve en la superficie de la cara (23)

El objetivo de este reporte es dar a conocer el manejo de un paciente con diagnóstico de quiste dentígero mandibular, para eliminar la patología y brindar condiciones de vida favorables al paciente.

\section{REPORTE DE CASO}

Paciente de sexo masculino, de 14 años, manifiesta que hace aproximadamente 5 meses, ha observado un abultamiento en la región nasogeniana del lado izquierdo de la cara. Al examen intraoral se observa expansión y adelgazamiento de la tabla vestibular.

\section{Exámenes complementarios.}

Radiografía panorámica (Figura 1A) .Tomografía Axial Computarizada (corte coronal de tercio facial inferior)(Figura 2B). Corte Sagital De Cuerpo Mandibular En Región Geniana (Figura 2).

Se observó una imagen radiolúcida entre las piezas dentales 42,44 y 45 con la pieza pieza $N^{\circ} 43$ incluida en la sínfisis de la región mandibular, posición horizontal de la pieza. 


\section{Diagnóstico. Quiste Dentígero}

Plan de tratamiento. Endodoncia de las piezas dentales $N^{\circ} 43,44,45$, enucleación de la lesión quística, exéresis del órgano dental $N^{\circ} 43$ curetaje y fijación ósea rígida.

Ejecución y evolución del tratamiento. Se realizó una profilaxis y el tratamiento endodóntico profiláctico de órganos dentales 43, 44 y 45 (Figura 1D).

En una siguiente cita se realizo la cirugía para la enucleación del quiste dentígero. Después de la asepsia y antisepsia correspondientes de la zona quirúrgica, se procedió con el bloqueo anestésico del nervio dentario inferior, lingual y bucal largo (Figura 2A), y del nervio mentoniano (Figura 2B), se empleo una solución anestésica de lidocaína al $2 \%$ con epinefrina 1:100 000 .

La incisión se realizo con una hoja $15 \mathrm{c}$ y mango de bisturí N 3, se profundizó hasta la cortical ósea, incidiendo en el periostio con extensión de la dental \#42 (Figura 2C). Se realizó un colgajo de espesor total, para la desinserción se utilizó una legra. Se sostuvo el colgajo con un separador de Minnesota, y se aplicó una ligera presión en el hueso para evitar la isquemia (Figura 2D). La osteotomía se realizó con una fresa quirúrgica redonda \#708 y con baja velocidad (Figura 2E).

Cuando estuvo expuesta la parte anterior de la mandíbula, se realizó la aspiración de la lesión, observándose un líquido color amarillento (Figura $2 \mathrm{~F}$ ) seguidamente se realizó la enucleación de la lesión(Figura 2G). Con una cureta de Lucas se eliminó todo el tejido de granulación. Se envió el tejido al anátomo-patológico, en concentración de formol 9:1 (9cc de solución fisiológica y $1 \mathrm{cc}$ de formol).

La cavidad remanente se lavó con abundante solución fisiológica para eliminar restos de tejido y proporcionar una zona ideal para la fijación ósea rígida con placas y tornillos de titanio (Figura $2 \mathrm{H}$ ).

distancia del órgano dental \#33 hasta órgano

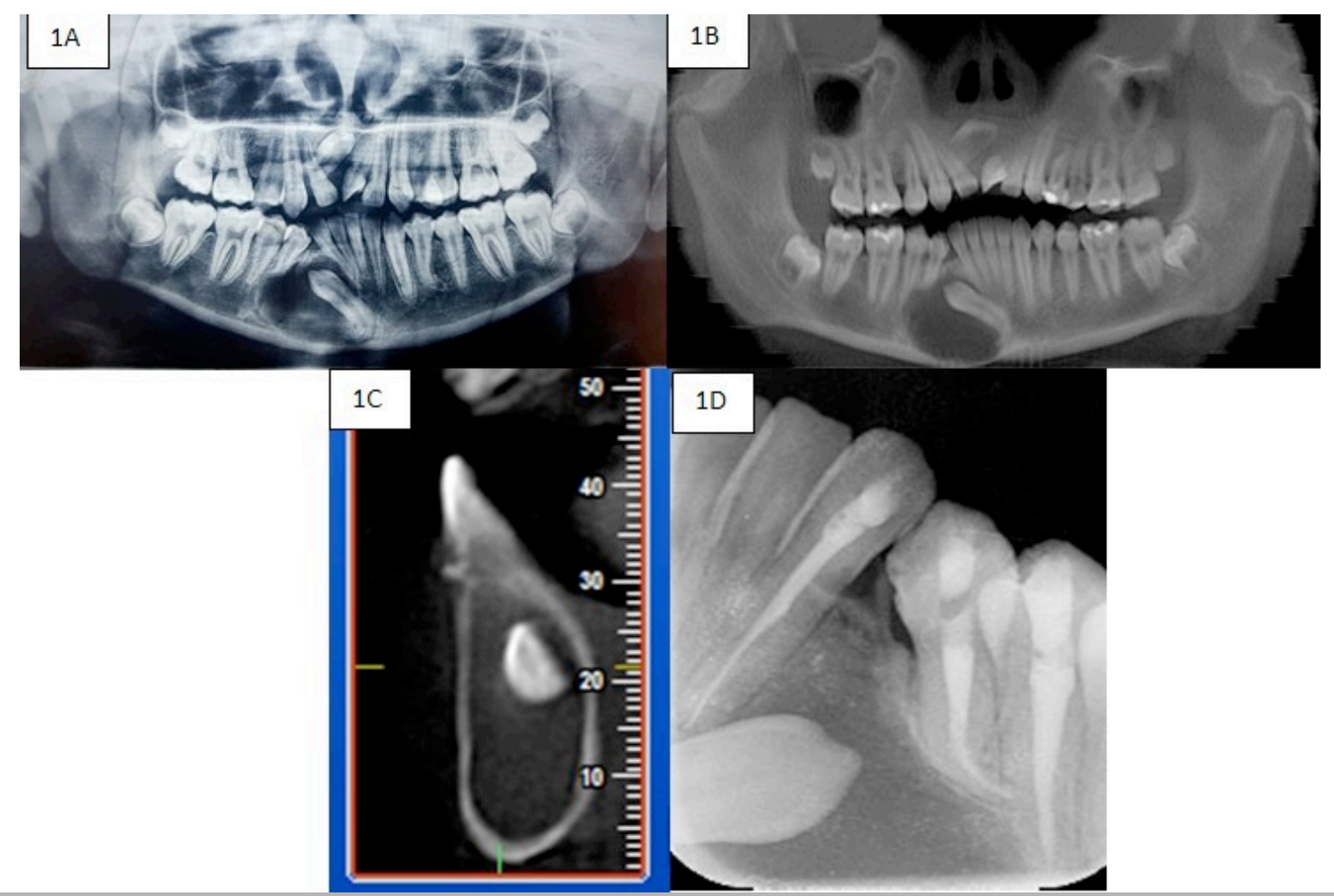

Figura 1. A.Radiografia Panorámica. B. Tomografía Axial Computarizada (corte coronal de tercio facial inferior). C. Corte Sagital De Cuerpo Mandibular En Región Geniana.D.Endodoncia de las piezas dentales 42, 44,45, 


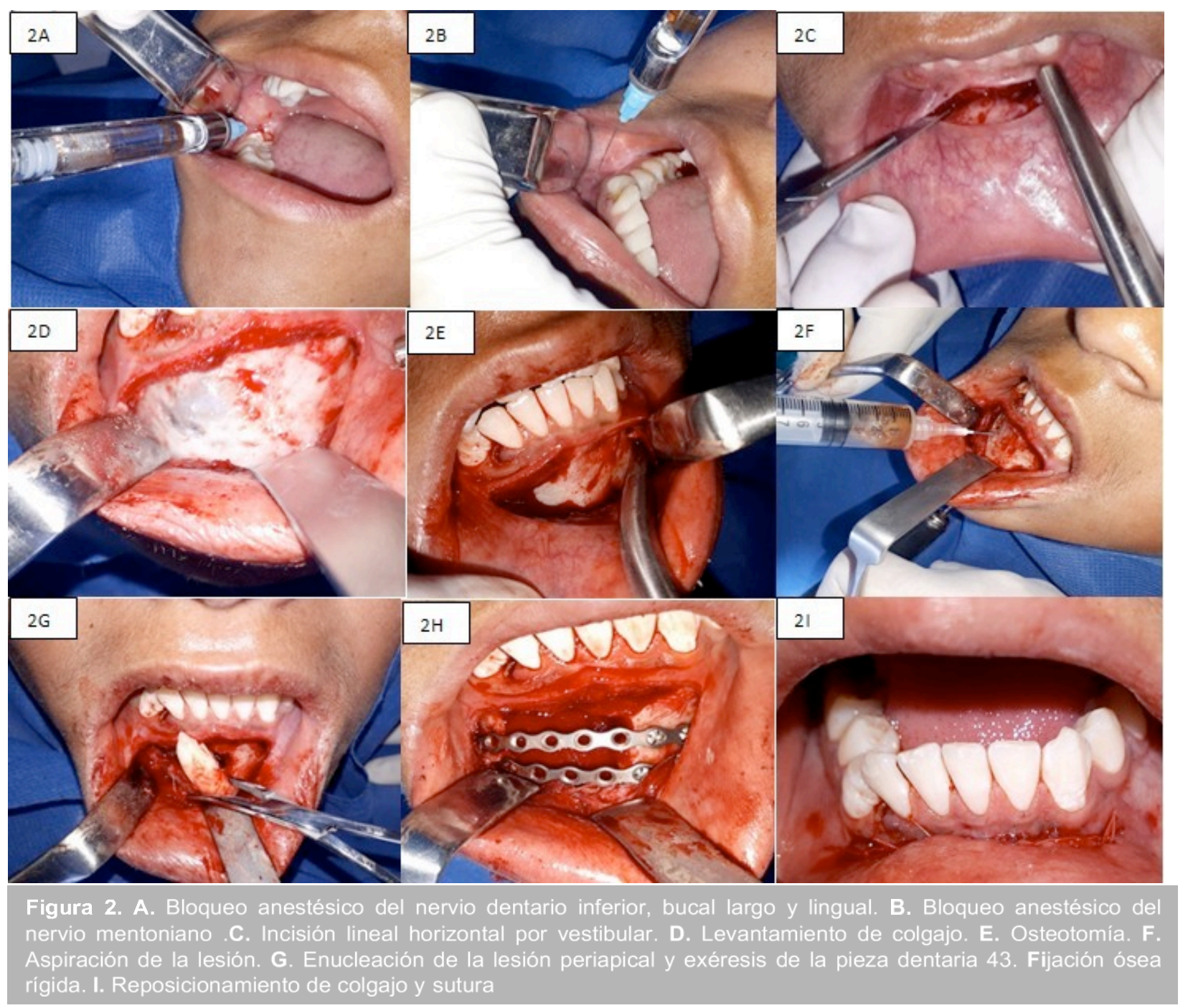

Una vez concluida esta fase del procedimiento se repuso el colgajo y se suturó con puntos continuos, se utilizo hilo de nylon 3 ceros (3-0). (Figura 2I). Terminada la sutura, se colocó una gasa estéril y se le pidió al paciente que ocluya para favorecer la formación del coágulo. La prescripción médica y cuidados postoperatorios se entregaron por escrito.

\section{Seguimiento del tratamiento ejecutado.}

Siete días después de la enucleación del quiste dentígero (Figura 3A), se retiraron las suturas (Figura 3B). Durante el control posoperatorio se observó clínicamente una cicatrización satisfactoria. Se le indicó al paciente podía continuar con su dieta normal y que debía acudir a las citas de control para controlar los resultados obtenidos, con la cirugía realizada.

\section{Evaluación Postoperatoria al mes.}

El paciente no manifestó síntomas. Al examen clínico no se observaron signos de recidiva de la lesión quística. Radiográficamente se observó una imagen radiopaca compatible aparentemente con regeneración del tejido óseo, la cual se veía separada del hueso basal original, por un tenue halo radiolúcido. (Figura $3 \mathrm{C}$ ). 


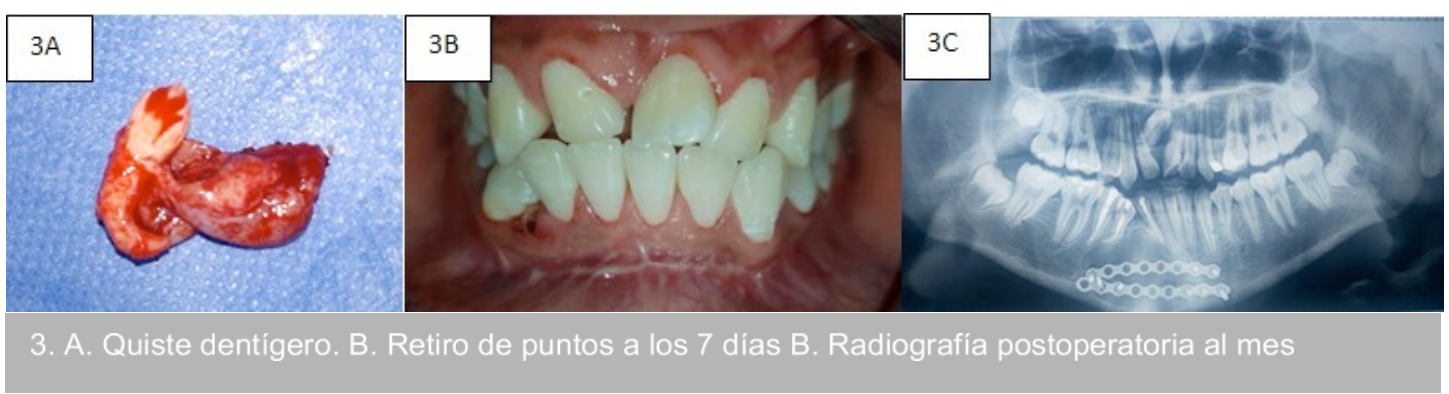

\section{DISCUSION}

El quiste dentígero es un quiste odontógenico muy común y se caracteriza por estar asociado a la corona de un diente no erupcionado ${ }^{(24)}$, es más prevalente en el sexo femenino y en personas mayores a 20 años (25); sin embargo, puede presentarse asociado a dientes supernumerarios en niños o adolescentes ${ }^{(26)}$. En este reporte se presenta el caso de un paciente masculino de catorce años de edad con un canino mandibular incluido, asociado a quiste dentígero. Es importante identificar y valorar una pieza retenida, porque puede derivar en diferentes tipos de alteraciones, como las asimetrías faciales o en ciertos síntomas manifestados por el paciente, que no tienen origen aparente alguno.

Se debe referir al paciente a estudios complementarios imagenológicos frente a la ausencia de órganos dentales porque nos brindará una proximidad al diagnóstico y se podrá elaborar un plan de tratamiento adecuado y evitar complicaciones.

A pesar de las diferentes técnicas de abordaje quirúrgico para esta patología, optamos por la enucleación total de la lesión a razón de que varios autores coinciden que los quistes foliculares pueden transformarse en tumores ${ }^{(26)}$, además en el presente caso se expone una cortical vestibular destruida por ende el desplazamiento mucoperiostal está indicado ya que esta cortical no se mantendrá después del tratamiento.

La enucleación constituye una técnica de tratamiento efectivo para la eliminación de la lesión que resultó ser benigna en los estudios histopatológicos y la fijación ósea rígida permitió una cicatrización ósea primaria.
Contribuciones de autoría: DXNS, KALLM, NACC, MJJP,JACP, Participaron en el diagnostico, tratamiento, revisión de la información, redacción y aprobación del artículo.

Fuente de financiamiento: Autofinanciado.

Conflictos de interés: No declararon conflictos de Interés

\section{REFERENCIAS}

1. Allais de Maurette M. Tratamiento de quiste dentígero bilateral mandibular por medio de dos tipos de tratamientos. Relato de caso clínico y comparación entre las técnicas. Acta Odontol Venez.2016; 45(1).

2. Rojas L, Claudio CA, Rojas GV. Clinical and Histopathological Study of Dentigerous Cyst Associated to Maxilar Lateral Tooth: Case Report. Int J of Dental Sciences. 2017.

3. Soolari A, Soolari A, Fielding C. Inflamed Odontogenic Cyst with Actinomyces Colonization: Management of an Atypical Case in a 16-Year-Old Patient. Int J Periodontics Restorative Dent. 2020; 40(2):35-41.

4. Shoaee S, Khazaei P, Mashhadiabbas F, Varshosaz M, Sharifi F, Hessarl H. Association between tooth impaction and odontogenic lesions: A matched case-control study. Med J Islam Repub Iran. 2016; 32:57.

5. Kammer PV, Mello FW, Rivero ERC. Comparative analysis between developmental and inflammatory odontogenic cysts: retrospective study and literature review. Oral Maxillofac Surg. 2019 [cited 2020 feb 20]; 24(1) Disponible https://www.ncbi.nlm.nih.gov/31858303.

6. Chen J, LV D, Li M, Zhao W, He Y. The correlation between the three-dimensional radiolucency area around the crown of impacted maxillary canines and dentigerous cysts. Dentomaxillofac Radiol. 2020 . 
7 Bassetti MA, Kuttenberger J, Novak J, Bassetti RG. The dentigerous cyst: two different treatment options illustrated by two cases. Swiss Dent J. 2019; 129(3):193-203.

8 Alnofaie $\mathrm{H}$, Alomran $\mathrm{O}$, Ababtain $\mathrm{R}$, Alomar A. Spontaneous Eruption of a Deeply Impacted Premolar After Conservative Treatment of an Associated Dentigerous Cyst: A Case Report. Cureus. 2019 [cited 2020 feb 20]; 18; 11(12) Disponible https://www.ncbi.nlm.nih.gov/6970100.

9 Martinelli-Kläy CP, Martinelli CR, Martinelli C, Macedo HR, Lombardi T. Unusual Imaging Features of Dentigerous Cyst: A Case Report. Dent J (Basel). 2019;7(3).

10 Mohammed M, Mahomed F, Ngwenya S. A survey of pathology specimens associatedWith impacted teeth over a 21-year period. Med Oral Patol Oral Cir Bucal. 2019; 24(5):571-576.

11 Patil AS, Jathar PN, Panse AM, Bahutule SR, Patil RU, Patil M. Infected Dentigerous Cyst and its Conservative Management: A Report of Two Cases. Int J Clin Pediatr Dent. 2019; 12(1):6872.

12 Weber M, Ries J, Büttner-Herold M, Geppert Cl, Kesting $M$, Wehrhan $F$. Differences in Inflammation and Bone Resorption between Apical Granulomas, Radicular Cysts, and Dentigerous Cysts. J Endod. 2019;45(10):12001208.

13 Karabas HC, Ozcan I, Tekkesin MS, Tasyapan SA, Guray B, Atapek MM. Evaluation of Radiolucent Lesions Associated with Impacted Teeth: A Retrospective Study. Curr Med Imaging Rev. 2020 [cited 2020 feb 20]; Disponible en: https://www.ncbi.nlm.nih.gov/32026783.

14 Rajendra Santosh AB. Odontogenic Cysts. Dent Clin North Am. 2020;64(1):105-119.

15 Taysi M, Ozdem C, Cankaya B, Yildirim S, Bilgic L. Conservative approach to a large dentigerous cyst in an 11-year-old patient. J Istanb Univ Fac Dent. 2016;50(3):51-56.

16 Sarmiento L, Robertson J, Ocampo A, Cepeda LA, Huerta E. Prevalence and distribution of odontogenic cysts in a Mexican sample. A 753 cases study. J Clin Exp Dent. 2017;9(4):e531e538.

17 Murakami K, Yamamoto K, Tsuyuki M, Sugiura T, Tsutsumi S, Kirita T. Theoretical efficacy of preventive measures for pathologic fracture after surgical removal of mandibular lesions based on a three-dimensional finite element analysis. J Oral Maxillofac Surg. 2014; 72(4):118.

18 Lovald S, Baack B, Gaball C, Olson G, Hoard A. Biomechanical optimization of bone plates used in rigid fixation of mandibular symphysis fractures. J Oral Maxillofac Surg. 2010; 68(8):1833-41.

19 De Moraes ATL, Soares HA, Viana Pinheiro JJ, Ribeiro Ribeiro AL. Marsupialization before enucleation as a treatment strategy for a large calcifying odontogenic cyst: Case report. Int J Surg Case Rep. 2020; 67:239-244.

20 Sgerdh AJ. Titanio o acero como material de osteosíntesis. El cirujano de accidentes. 2017; 120(2).

21 Kazi AA, Lee TS, Vincent A, Sokoya M, Sheen $D$, Ducic $Y$. The Role of External Fixation in Trauma and Reconstruction of the Mandible in the Age of Rigid Fixation. Facial Plast Surg. 2019; 35(6):614-622.

22 Pham Dang N, Barthélémy I, Bekara F. From rigid bone plate fixation to stable dynamic osteosynthesis in mandibular and craniomaxillo- facial surgery: Historical evolution of concepts and technical developments. J Stomatol Oral Maxillofac Surg. 2019; 120(3):229-233.

23 Yeoh M, Cunningham LL Jr. Concepts of Rigid Fixation in Facial Fractures.Atlas Oral Maxillofac Surg Clin North Am. 2019; 27(2):107-112.

24 Jendi SK, Shaikh S. El diente cruzando el encierro de la mandíbula: un único Expresión de la variedad central de quiste dentígero. Indian J Otolaryngol Head Neck Surg. 2019; 71: 860-864.

25 Sindi AM. Bilatteral Mandibular dentigerous. Cysts Presenting as an Incidental Finding: A case report. An J Case Rep. 2019 Aug 5;20:1148-1159.

26 Rudraraju A, Venigalla A, Babburi S, Soujanya $P$, Subramanyam RV, Lakshmi KR.Calretinin expression in odontogenic cysts and odontogenic tumors and thepossible role of calretinin in pathogenesis of ameloblastoma. J Oral Maxillofac Pathol. 2019; 23(3):349-355

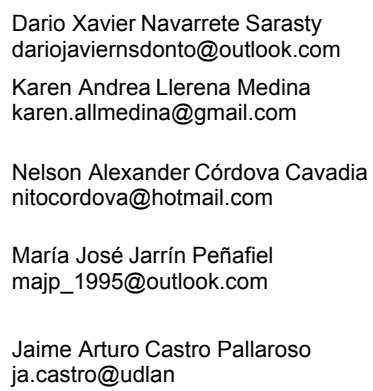

https://orcid.org/0000-0001-6044-5851

https://orcid.org/0000-0001-9115-4512

https://orcid.org/0000-0003-0276-0356

https://orcid.org/0000-0002-1875-1254

https://orcid.org/ 0000-0002-3477-6953 